\title{
THE EFFECT OF SURFACE TENSION IN POROUS WAVE MAKER PROBLEMS
}

\author{
A. CHAKRABARTI ${ }^{1}$ and T. SAHOO ${ }^{1}$
}

(Received 13 October 1995; revised 31 January 1997)

\begin{abstract}
Using a mixed-type Fourier transform of a general form in the case of water of infinite depth and the method of eigenfunction expansion in the case of water of finite depth, several boundary-value problems involving the propagation and scattering of time harmonic surface water waves by vertical porous walls have been fully investigated, taking into account the effect of surface tension also. Known results are recovered either directly or as particular cases of the general problems under consideration.
\end{abstract}

\section{Introduction}

The problems of propagation of surface water waves due to a vertical wave maker have been treated by various researchers using the classical wave maker theory developed by Havelock [6]. This wave maker theory has been further generalised by Rhodes-Robinson [12] when the effect of surface tension is taken into account. The complications of handling this more general class of problems lie in the fact that a third-order derivative appears, in a natural way, in the mathematical description of one of the boundary conditions, whereas the basic partial differential equation that is required to be solved is Laplace's equation, in two dimensions. Rhodes-Robinson [12] has devised a method based on the Green's integral theorem to solve the boundaryvalue problem of concern. Later on, Rhodes-Robinson [13] employed the reduction technique of Williams [18] to solve more general boundary-value problems of this class. Mandal and Chakrabarti [11] devised a method based on the Fourier cosine transform to solve the wave maker problem of Havelock [6]. Mandal and Bandyopadhyay [10] generalised the problem of Mandal and Chakrabarti [11] with the extra effect of surface tension. In these works the wave maker has been represented by a vertical impermeable wall oscillating horizontally.

${ }^{1}$ Department of Mathematics, Indian Institute of Science, Bangalore 560 012, India

(C) Australian Mathematical Society, 1998, Serial-fee code 0334-2700/98 
It was pointed out by Madsen [9] that the leakage effect will be large in reducing the wave amplitude. Chwang [4] developed a porous wave maker theory to investigate the porous effect of a wave maker in the case of water of finite depth and Chakrabarti [1] generalised Chwang's study with the extra effect of surface tension by using a mixed type of Fourier transform. Currently Gorgui, Faltas and Ahmed [5] have studied the problem of capillary gravity waves in the presence of an infinite porous wall, by using Green's integral theorem.

Recently Chakrabarti and Sahoo [2] have studied the reflection of water waves by a nearly vertical porous wall, by using a mixed type of Fourier transform similar to that utilised by Chakrabarti [1] earlier.

The amplitude and the frequency of the waves depend on both the surface tension and gravity. In most cases, like oceans, lakes and reservoirs, the effect of surface tension can be neglected. But while doing experimental study it may not be possible to neglect the effect of surface tension. As mentioned by Hocking and Mahdmina [8], another important reason for including surface tension is that in the absence of surface tension the transient motion initiated by an impulsive start is singular, but when the effect of surface tension is taken into account this singularity is removed.

In the present study we have investigated the effect of surface tension on a piston type porous wave maker having fine pores on it, in the case of water of infinite as well as finite depths. Because of the effect of surface tension, the uniqueness of the solution of the problems depend on the behaviour of a special combination of the derivatives of the velocity potential at the edge, that is, at the points $(0 \pm, 0)$, which is still a topic of research. In the case of impermeable obstacles like surface-piercing vertical barriers or a wall, Hocking [7] gave a more acceptable edge condition which relates the slope of the contact line to the wave maker. As far as permeable obstacles are concerned no valid results are available in the literature. In our present work, we will use an edge condition which is similar to that of Hocking [7] with certain modifications as suggested by Rhodes-Robinson [17]. In the case of water of infinite depth, we have reinvestigated the results of Gorgui, Faltas and Ahmed [5], by using a general mixed transform of Fourier type. In the case of water of finite depth, we have used the eigenfunction expansion method as has been discussed by Rhodes-Robinson [14] in a different investigation.

As an application of the one-sided porous wave maker problem, the full solution of the boundary-value problem related to the reflection of water waves by a porous wall in the case of water of finite depth is derived. The full solution in the case of the problem of reflection of water waves by the porous wall is obtained by subtracting out the incident waves from the velocity potential to obtain a special case of the one-sided wave maker problem as discussed in Section 5. As an application of the two-sided wave maker problem as well as the problem of reflection of water waves by a porous wall, the problem of scattering of water waves by a vertical porous plate in the case of 
water of finite depth is studied by way of obtaining the full solution of the boundaryvalue problem associated with it. The full solution in the case of the scattering problem is derived by reducing the boundary-value problem to two boundary-value problems, which are particular cases of the problem of reflection of water waves by the porous wall. The one-sided porous wave maker problem is expected to have applications in the study of surface waves in rock filled dams and land slides during earthquakes in water reservoirs and lakes. Sometimes it is needed to reduce the wave amplitude by using certain kind of porous devices. The two-sided porous wave maker problem has applications in cases where generation of surface waves is of main interest. Since the velocity potential is known completely, the pressure difference along the two sides of the plate, the surface elevation and the other important physical quantities can be determined easily by using the final solution, once the edge slope constant for the material is known completely. Certain numerical results involving the reflection coefficients in the case of reflection of water waves by a vertical porous wall as well as the reflection and transmission coefficients in the case of scattering of water waves by a vertical porous plate, have been obtained in the case of finite depth of the fluid. These numerical results have been presented in the forms of graphs (see Figures 1, 2,3).

In the present study we have assumed the wall to be made of materials with fine pores.

\section{Waves generated by the piston type two-sided porous wave maker}

The problem under consideration is two dimensional in nature. We use a rectangular Cartesian co-ordinate system $(x, y)$ in which the $x$-axis extends horizontally and $y$-axis is taken in the vertically downward direction. A piston type porous wave maker made up of fine pores occupies the position $x=0$, in the semi-infinite fluid region $y>0$. This wave maker is assumed to oscillate with the velocity $\operatorname{Re}\left(U(y) e^{-i \omega t}\right)$. Assuming that the fluid is incompressible and inviscid and the motion is irrotational and simple harmonic in time with angular frequency $\omega$, we have the existence of the velocity potential $\Phi_{j}(x, y, t)=\mathfrak{R}\left\{\phi_{j}(x, y) e^{-i \omega t}\right\}$, ' $\mathfrak{R}$ ' denoting the real part. The functions $\phi_{j}$ for $j=1,2$ represent respectively the velocity potentials for $x>0$ and $x<0$ respectively. We study the effect of surface tension on the wave maker in both the cases of water of infinite and finite depths by way of determining the velocity potentials $\phi_{j}(x, y)$, after dropping the factor $e^{-i \omega t}$ from the whole mathematical formulation of the physical problem, which satisfies the partial differential equation

$$
\nabla^{2} \phi_{j}=0 \text { in the fluid region }
$$

subject to the free surface boundary conditions

$$
M \phi_{j y y y}+\phi_{j y}+K \phi_{j}=0 \quad \text { on } \quad y=0,
$$


where $K=\omega^{2} / g, M=T /(\rho g)$ have their usual meaning (see Rhodes-Robinson [16]). On the wave maker (see Chwang [4]), we have that

$$
\phi_{j x}=-i k\left(\phi_{1}-\phi_{2}\right)+U(y), \quad \text { on } \quad x=0,
$$

where $k=b \rho \omega / \mu, \mu$ is the dynamic viscosity, $\rho$ is the constant density of the fluid.

The conditions at infinity are

$$
\begin{array}{ll}
\phi_{1} \rightarrow R_{1} e^{-k_{0} y+i k_{0} x} & \text { as } \quad x \rightarrow \infty, \\
\phi_{2} \rightarrow R_{2} e^{-k_{0} y-i k_{0} x} & \text { as } \quad x \rightarrow-\infty, \\
\phi_{j},\left|\nabla \phi_{j}\right| \rightarrow 0 & \text { as } \quad y \rightarrow \infty,
\end{array}
$$

in case of water of infinite depth where $R_{1}$ and $R_{2}$ are unknown constants to be determined and $k_{0}$ is the real positive root of the cubic equation $\alpha\left(1+M \alpha^{2}\right)=K$, in $\alpha$.

Whereas, in the case of water of finite depth, the infinity and the bottom conditions are

$$
\begin{array}{ll}
\phi_{1} \rightarrow A_{0} \cosh k_{0}(h-y) e^{i k_{0} x} & \text { as } \quad x \rightarrow \infty, \\
\phi_{2} \rightarrow B_{0} \cosh k_{0}(h-y) e^{-i k_{0} x} & \text { as } \quad x \rightarrow-\infty,
\end{array}
$$

and

$$
\phi_{j y}=0 \quad \text { on } y=h,
$$

where $y=h$ is the rigid bottom surface and $k_{0}$ is the solution of the transcendental equation $k_{0}\left(1+M k_{0}^{2}\right) \sinh k_{0} h=K \cosh k_{0} h$.

Further for the uniqueness of the solution of the boundary-value problems under consideration, we have to meet with the generalized edge conditions (see RhodesRobinson [17])

$$
\begin{aligned}
& \phi_{1 x y}(0+, 0)+i k\left(\phi_{1 y}(0,0)-\phi_{2 y}(0,0)\right)=\pi \lambda_{0}^{+}, \\
& \phi_{2 x y}(0-, 0)+i k\left(\phi_{1 y}(0,0)-\phi_{2 y}(0,0)\right)=\pi \lambda_{0}^{-},
\end{aligned}
$$

where $\lambda_{0}^{+}, \lambda_{0}^{-}$are indeterminate in nature and would be determined from the dynamical edge conditions.

\section{Method of solution for the case of water of infinite depth}

We set

$$
\begin{aligned}
& \phi_{1}=\psi_{1}+R_{1} e^{-k_{0} y+i k_{0} x}, \\
& \phi_{2}=\psi_{2}+R_{2} e^{-k_{0} y-i k_{0} x}
\end{aligned}
$$


and define a Fourier type integral transform $\hat{\psi}_{j}(x, \xi)$ for the function $\psi_{j}(x, y)$ as given by the relation

$$
\hat{\psi}_{j}(x, \xi)=\int_{0}^{\infty} L(\xi, y) \psi_{j}(x, y) d y-\xi M \psi_{j y}(x, 0), \quad \xi>0,
$$

where

$$
L(\xi, y)=\xi\left(1-M \xi^{2}\right) \cos \xi y-K \sin \xi y
$$

The expression (3.1) for $\hat{\psi}_{j}(x, \xi)$ can be rewritten after integrating by parts as

$$
\hat{\psi}_{j}(x, \xi)=-\int_{0}^{\infty}\left\{M \frac{\partial^{3}}{\partial y^{3}}+\frac{\partial}{\partial y}+K\right\} \psi_{j}(x, y) \sin \xi y d y .
$$

Using the Fourier sine inversion to the relation (3.5) we obtain that

$$
\begin{aligned}
\psi_{j}(x, y)= & C_{1 j}(x) e^{-k_{0} y}+C_{2 j}(x) e^{-\omega_{1} y}+C_{3 j}(x) e^{-\omega_{2} y} \\
& +\frac{2}{\pi} \int_{0}^{\infty} \frac{L(\xi, y)}{\xi^{2}\left(1-M \xi^{2}\right)^{2}+K^{2}} \hat{\psi}_{j}(x, \xi) d \xi
\end{aligned}
$$

where $C_{1 j}(x), C_{2 j}(x), C_{3 j}(x)$ are arbitrary functions of $x$ and $k_{0}, \omega_{1}$ and $\omega_{2}$ are the roots of the cubic equation $\alpha\left(1+M \alpha^{2}\right)=K, k_{0}$ is real and positive, $\omega_{1}$ and $\omega_{2}$ are the complex roots with negative real parts. Because of the behaviour of $\psi_{j}(x, y)$ as $y \rightarrow \infty$ we must have

$$
C_{2 j}(x)=C_{3 j}(x)=0 .
$$

Substituting for $\phi_{j}$ from (3.1) and (3.2) and using the mixed transform to the equations (2.1) along with the boundary conditions (2.2) and (2.4) we obtain that $\hat{\psi}_{j}(x, \xi)$ satisfy the ordinary differential equations

$$
\hat{\psi}_{j}^{\prime \prime}-\xi^{2} \hat{\psi}_{j}=0, \quad(j=1,2),
$$

prime denoting ordinary derivative with respect to $x$ (note that $\xi$ is just a transform parameter), with the infinity condition as given by

$$
\hat{\psi}_{j}(x, \xi) \rightarrow 0 \quad \text { as } \quad|x| \rightarrow \infty
$$

The solutions of the equations (3.8) satisfying the conditions (3.9) are given by

$$
\hat{\psi}_{j}(x, \xi)=A_{j}(\xi) e^{-\xi|x|}, \quad(j=1,2)
$$

where $A_{j}(\xi)$ are determined as described below. 
Substituting for $\hat{\psi}_{j}(x, \xi)$ in the expression (3.6) we obtain that

$$
\psi_{j}(x, y)=C_{1 j}(x) e^{-k_{0} y}+\frac{2}{\pi} \int_{0}^{\infty} \frac{L(\xi, y)}{\xi^{2}\left(1-M \xi^{2}\right)^{2}+K^{2}} A_{j}(\xi) e^{-\xi|x|} d \xi
$$

Again using the fact that $\psi_{j}(x, y)$ 's satisfy the Laplace equation with $\psi_{j}(x, y) \rightarrow 0$ as $|x| \rightarrow \infty$ we obtain that

$$
C_{1 j}(x)=0 .
$$

Thus, from the relations (3.1), (3.2), (3.11) and (3.12), we derive that

$$
\begin{aligned}
& \phi_{1}(x, y)=R_{1} e^{-k_{0} y+i k_{0} x}+\frac{2}{\pi} \int_{0}^{\infty} \frac{L(\xi, y)}{\xi^{2}\left(1-M \xi^{2}\right)^{2}+K^{2}} A_{1}(\xi) e^{-\xi x} d \xi \\
& \phi_{2}(x, y)=R_{2} e^{-k_{0} y-i k_{0} x}+\frac{2}{\pi} \int_{0}^{\infty} \frac{L(\xi, y)}{\xi^{2}\left(1-M \xi^{2}\right)^{2}+K^{2}} A_{2}(\xi) e^{\xi x} d \xi
\end{aligned}
$$

From the conditions (2.3) we have that

$$
\phi_{1 x}=\phi_{2 x} \quad \text { on } \quad x=0, \quad y>0,
$$

which, after substituting for $\phi_{1}(x, y)$ and $\phi_{2}(x, y)$ from the relations (3.13) and (3.14), gives that (see Rhodes-Robinson [12])

$$
\begin{array}{ll} 
& A_{1}(\xi)+A_{2}(\xi)=C, \\
\text { and } & \\
& R_{1}+R_{2}=2 C i /\left(1+3 M k_{0}^{2}\right),
\end{array}
$$

where $C$ is an arbitrary constant.

In the present study, we will assume that the motion is antisymmetric and hence $C=0$. Thus the full solution will be obtained by determining $A_{1}(\xi), A_{2}(\xi), R_{1}$ and $R_{2}$.

Substituting for $\phi_{1}$ and $\phi_{2}$ in the relation (2.3) from the expressions (3.13) and (3.14) after making use of the results (3.16) with the assumption $C=0$, we obtain that

$$
i R_{1}\left(k_{0}+2 k\right) e^{-k_{0} y}-\frac{2}{\pi} \int_{0}^{\infty} \frac{(\xi-2 i k) A_{1}(\xi) L(\xi, y) d \xi}{\xi^{2}\left(1-M \xi^{2}\right)^{2}+K^{2}}=U(y) .
$$

Considering the mixed transform of the relation (3.17), and making use of the result (2.6), we obtain that

$$
A_{1}(\xi)=-\left\{\int_{0}^{\infty} U(y) L(\xi, y) d y-\xi M \pi \lambda_{0}^{+}\right\} /(\xi-2 i k) .
$$


In a similar way from the conditions (2.3) and making use of the result (2.6), we obtain that

$$
A_{2}(\xi)=\left\{\int_{0}^{\infty} U(y) L(\xi, y) d y-\xi M \pi \lambda_{0}^{-}\right\} /(\xi-2 i k)
$$

From the relations (3.16), (3.18) and (3.19) with $C=0$ we obtain that

$$
\lambda_{0}^{+}=\lambda_{0}^{-},
$$

which suggests that the plate is of same material on both sides.

Next, to determine $R_{1}$, as the motion is anti symmetric, we have

$$
\phi_{1}(0, y)=-\phi_{2}(0, y)
$$

From the expression (3.13) we derive that

$$
\phi_{1 y}(x, 0)=-k_{0} R_{1} e^{i k_{0} x}-\frac{2 K}{\pi} \int_{0}^{\infty} \frac{\xi A_{1}(\xi) e^{-\xi x} d \xi}{\xi^{2}\left(1-M \xi^{2}\right)^{2}+K^{2}}
$$

and

$$
\int_{0}^{\infty} \phi_{1}(x, y) e^{-k_{0} y} d y=\frac{R_{1} e^{-i k_{0} x}}{2 k_{0}}-\frac{2 M k_{0}}{\pi} \int_{0}^{\infty} \frac{\xi A_{1}(\xi) e^{-\xi x} d \xi}{\xi^{2}\left(1-M \xi^{2}\right)^{2}+K^{2}}
$$

so that, after eliminating the integral, we obtain

$$
\frac{\phi_{1 y}(x, 0)+k_{0} R_{1} e^{i k_{0} x}}{K}=\frac{\int_{0}^{\infty} \phi_{1}(x, y) e^{-k_{0} y} d y-R_{1} e^{i k_{0} x} /\left(2 k_{0}\right)}{M k_{0}} .
$$

Operating both sides of the relation (3.24) by $\frac{\partial}{\partial x}+2 i k$ and making use of the conditions (2.3) and (2.6), we obtain, after taking limit as $x \rightarrow 0+$, that

$$
R_{1}=\frac{-2 i\left\{K a\left(k_{0}\right)-M k_{0} \pi \lambda_{0}^{+}\right\}}{\left(1+3 M k_{0}^{2}\right)\left(k_{0}+2 k\right)},
$$

where $a\left(k_{0}\right)=\int_{0}^{\infty} U(s) e^{-k_{0} s} d s$. Substituting for $R_{1}$ in the relation $R_{1}=-R_{2}$, we can obtain $R_{2}$. Thus the full solution in this case is obtained completely, when the relations (3.13), (3.14), (3.18)-(3.20) and (3.25) are utilized. 


\section{Method of solution for the case of water of finite depth}

In this case, using the method of separation of variables, the solution of the boundary-value problems for $\phi_{1}$ and $\phi_{2}$ satisfying the conditions (2.1), (2.2) and (2.5) have the form

$$
\begin{aligned}
& \phi_{1}(x, y)=A_{0} \cosh k_{0}(h-y) e^{i k_{0} x}+\sum_{n=1}^{\infty} A_{n} \cos k_{n}(h-y) e^{-k_{n} x}, \quad x>0, \\
& \phi_{2}(x, y)=B_{0} \cosh k_{0}(h-y) e^{-i k_{0} x}+\sum_{n=1}^{\infty} B_{n} \cos k_{n}(h-y) e^{k_{n} x}, \quad x<0,
\end{aligned}
$$

where $A_{n}, B_{n}$ are arbitrary constants which have to be determined and $k_{n}$ 's are the infinite number of roots of the transcendental equation

$$
k_{n}\left(1-M k_{n}^{2}\right) \sin k_{n} h+K \cos k_{n} h=0 .
$$

From (2.3) we have

$$
\phi_{1 x}=\phi_{2 x} \quad \text { on } \quad x=0 .
$$

Substituting for $\phi_{1}$ and $\phi_{2}$ from (4.1) and (4.2) in (4.3), we obtain that

$$
i k_{0}\left(A_{0}+B_{0}\right) \cosh k_{0}(h-y)-\sum_{n=1}^{\infty} k_{n}\left(A_{n}+B_{n}\right) \cos k_{n}(h-y)=0 \quad \text { for } 0<y<h .
$$

The full solution of the boundary-value problem will be determined, in this case, provided the $A_{n}$ 's and $B_{n}$ 's are completely determined.

Let us define the inner product

$$
\left\langle f_{n}, f_{m}\right\rangle=\int_{0}^{h} f_{m}(y) f_{n}(y) d y+\frac{M}{K} f_{n}^{\prime}(0) f_{m}^{\prime}(0), \quad \text { for } m, n=0,1,2, \ldots,
$$

where $f_{0}(y)=\cosh k_{0}(h-y), 0<y<h$, and $f_{n}(y)=\cos k_{n}(h-y), 0<y<h$, $n=1,2, \ldots$. Then we have that

$$
\int_{0}^{h} f_{0}(y) f_{n}(y) d y=\frac{M}{K} k_{0} k_{n} \sinh k_{0} h \sin k_{n} h, \quad n=1,2, \ldots,
$$

and

$$
\int_{0}^{h} f_{m}(y) f_{n}(y) d y=\frac{M}{K} k_{m} k_{n} \sin k_{m} h \sin k_{n} h, \quad \text { for } m \neq n m, n=1,2, \ldots
$$


Again

$$
\begin{aligned}
& f_{m}^{\prime}(0) f_{n}^{\prime}(0)=-k_{m} k_{n} \sin k_{m} h \sin k_{n} h, \quad \text { for } \quad m \neq n, m, n=1,2, \ldots, \\
& f_{0}^{\prime}(0) f_{n}^{\prime}(0)=-k_{0} k_{n} \sinh k_{0} h \sin k_{n} h, \quad \text { for } \quad n=1,2, \ldots
\end{aligned}
$$

From the relations (4.6)-(4.9) we thus have

$$
\left\langle f_{m}, f_{n}\right\rangle=0, \quad \text { for } m \neq n, m, n=1,2, \ldots,
$$

which suggests that the set of functions $\left\{f_{0}, f_{1}, \ldots\right\}$ are orthogonal with respect to the inner product as defined by the relation (4.5). It is assumed that the above set of functions forms a complete set (for details see Rhodes-Robinson [14]).

Then, by using the orthogonality property satisfied by the functions $f_{n}$ 's for $n=$ $0,1,2, \ldots$, we obtain from the relations (4.4) that

$$
\begin{aligned}
A_{0}+B_{0} & =\frac{4 k_{0} M i C_{1}\left(1+M k_{0}^{2}\right) \sinh k_{0} h}{K\left\{2 k_{0} h\left(1+M k_{0}^{2}\right)+\left(1+3 M k_{0}^{2}\right) \sinh 2 k_{0} h\right\}}, \\
A_{n}+B_{n} & =\frac{4 k_{n} M\left(1-M k_{n}^{2}\right) i C_{1} \sin k_{n} h}{K\left\{2 k_{n} h\left(1-M k_{n}^{2}\right)+\left(1-3 M k_{n}^{2}\right) \sin 2 k_{n} h\right\}},
\end{aligned}
$$

$$
\text { for } n=1,2, \ldots \text {, }
$$

where $C_{1}$ is an arbitrary constant. As in the case of water of infinite depth, we will assume that the motion is antisymmetric and hence $C_{1}=0$ in (4.11).

Again, we have

$$
\left\langle f_{0}, f_{0}\right\rangle=\frac{2 k_{0} h\left(1+M k_{0}^{2}\right)+\left(1+3 M k_{0}^{2}\right) \sinh 2 k_{0} h}{4 k_{0}\left(1+M k_{0}^{2}\right)}
$$

and

$$
\left\langle f_{n}, f_{n}\right\rangle=\frac{2 k_{n} h\left(1-M k_{n}^{2}\right)+\left(1-3 M k_{n}^{2}\right) \sin 2 k_{n} h}{4 k_{n}\left(1-M k_{n}^{2}\right)}, \quad n=1,2, \ldots
$$

Further, substituting for $\phi_{1}$ and $\phi_{2}$ from (4.1) and (4.2) in (2.3) after using (4.11) with $C_{1}=0$, we obtain that

$$
i A_{0}\left(k_{0}+2 k\right) f_{0}(y)-\sum_{n=1}^{\infty}\left(k_{n}-2 i k\right) A_{n} f_{n}(y)=U(y) .
$$

Taking the inner product with $f_{0}(y)$ we get

$$
A_{0}=-\frac{4 i k_{0}\left(1+M k_{0}^{2}\right)\left[\int_{0}^{h} U(s) \cosh k_{0}(h-s) d s-\frac{M k_{0}}{K} \pi \lambda_{0}^{+} \sinh k_{0} h\right]}{\left(k_{0}+2 k\right)\left\{2 k_{0} h\left(1+M k_{0}^{2}\right)+\left(1+3 M k_{0}^{2}\right) \sinh 2 k_{0} h\right\}}
$$


and

$$
B_{0}=\frac{4 i k_{0}\left(1+M k_{0}^{2}\right)\left[\int_{0}^{h} U(s) \cosh k_{0}(h-s) d s-\frac{M k_{0}}{K} \pi \lambda_{0}^{-} \sinh k_{0} h\right]}{\left(k_{0}+2 k\right)\left\{2 k_{0} h\left(1+M k_{0}^{2}\right)+\left(1+3 M k_{0}^{2}\right) \sinh 2 k_{0} h\right\}} .
$$

Using (4.11), (4.15) and (4.16) with $C_{1}=0$, we obtain

$$
\lambda_{0}^{+}=\lambda_{0}^{-},
$$

which suggest that the wave maker is made up of the same material on both sides.

Further, from (4.14), taking the inner product with $f_{n}(y)$, we obtain that

$$
A_{n}=\frac{4 k_{n}\left(1-M k_{n}^{2}\right)\left[\int_{0}^{h} U(s) \cos k_{n}(h-s) d s-\frac{M k_{n}}{K} \pi \lambda_{0}^{+} \sin k_{n} h\right]}{\left(k_{n}-2 i k\right)\left\{2 k_{n} h\left(1-M k_{n}^{2}\right)+\left(1-3 M k_{n}^{2}\right) \sin 2 k_{n} h\right\}} .
$$

Hence $B_{n}$ can be determined from (4.11), (4.17) and (4.18). Substituting for $A_{0}, B_{0}, A_{n}$ and $B_{n}$ in (4.1) and (4.2) we obtain the complete solution in this case.

\section{Waves generated by the one-sided porous wave maker}

In this case we are interested in the generation of surface waves only in the direction of positive $x$-axis. In such circumstances the potential function $\phi(x, y)$ satisfies the equation (2.1) with the boundary condition (2.2). The conditions (2.3) are replaced by

$$
\phi_{x}+i k \phi=U(y) \quad \text { on } \quad x=0, y>0,
$$

(This condition was derived in detail in Chakrabarti and Sahoo [2].) The infinity conditions are

$$
\phi \rightarrow R e^{-k_{0} y+i k_{0} x}, \quad \text { as } \quad x \rightarrow \infty
$$

and

$$
\phi,|\nabla \phi| \rightarrow 0 \quad \text { as } \quad y \rightarrow \infty
$$

in case of water of infinite depth where $R$ is an unknown constant to be determined.

In the case of water of finite depth, the conditions to be meet with are

$$
\phi \rightarrow A_{0} \cosh k_{0}(h-y) e^{i k_{0} x} \quad \text { as } \quad x \rightarrow \infty
$$


and

$$
\phi_{y}=0 \text { on } y=h .
$$

Finally the edge condition of the problems are given by

$$
\phi_{x y}(0+, 0)+i k \phi_{y}(0,0)=\pi \lambda
$$

where $\lambda$ is another undeterminable generalized edge slope constant (see Chakrabarti and Sahoo [3] and Rhodes-Robinson [17], where this condition was suggested).

Proceeding as in Section 3 for water of infinite depth and as in Section 4 for water of finite depth, we obtain that

$$
\phi(x, y)=R e^{-k_{0} y+i k_{0} x}+\frac{2}{\pi} \int_{0}^{\infty} \frac{L(\xi, y)}{\xi^{2}\left(1-M \xi^{2}\right)^{2}+K^{2}} A(\xi) e^{-\xi x} d \xi .
$$

Here

$$
\begin{gathered}
A(\xi)=-\left\{\int_{0}^{\infty} U(y) L(\xi, y) d y-\xi M \pi \lambda\right\} /(\xi-i k), \\
R=\frac{-2 i\left\{K a\left(k_{0}\right)-M k_{0} \pi \lambda\right\}}{\left(1+3 M k_{0}^{2}\right)\left(k_{0}+k\right)}
\end{gathered}
$$

in case of water of infinite depth, where $a\left(k_{0}\right)$ is as defined in (3.25) and

$$
\phi(x, y)=A_{0} \cosh k_{0}(h-y) e^{i k_{0} x}+\sum_{n=1}^{\infty} A_{n} \cos k_{n}(h-y) e^{-k_{n} x}, \quad x>0,
$$

in case of water of finite depth, where

$$
A_{0}=-\frac{4 i k_{0}\left(1+M k_{0}^{2}\right)\left[\int_{0}^{h} U(s) \cosh k_{0}(h-s) d s-M k_{0} / K \pi \lambda \sinh k_{0} h\right]}{\left(k_{0}+k\right)\left\{2 k_{0} h\left(1+M k_{0}^{2}\right)+\left(1+3 M k_{0}^{2}\right) \sinh 2 k_{0} h\right\}}
$$

and

$$
A_{n}=-\frac{4 k_{n}\left(1-M k_{n}^{2}\right)\left[\int_{0}^{h} U(s) \cos k_{n}(h-s) d s+M k_{n} / K \pi \lambda \sin k_{n} h\right]}{\left(k_{n}-i k\right)\left\{2 k_{n} h\left(1-M k_{n}^{2}\right)+\left(1-3 M k_{n}^{2}\right) \sin 2 k_{n} h\right\}} .
$$




\section{Reflection of water waves by a vertical porous wall}

As an application of the solutions obtained in Section 5, let us consider the reflection of water waves by a vertical porous wall. The case of water of infinite depth has been investigated by Rhodes-Robinson [17] and Chakrabarti and Sahoo [3]. Here, we will only consider the case of water of finite depth.

In this case the conditions (5.1), (5.4) are replaced by

$$
\phi_{x}+i k \phi=0 \text { on } x=0,0<y<h
$$

and

$$
\phi \rightarrow \cosh k_{0}(h-y)\left\{e^{-i k_{0} x}+A_{0} e^{i k_{0} x}\right\} \quad \text { as } \quad x \rightarrow \infty,
$$

where $A_{0}$ is the unknown reflection coefficient to be determined.

Setting $\phi(x, y)=\psi(x, y)+e^{-i k_{0} x} \cosh k_{0}(h-y)$, in the boundary-value problem for $\phi(x, y)$, a problem for $\psi(x, y)$ is obtained which is a particular case of the wave maker problem considered in Section 5, with $U(y)=i\left(k_{0}-k\right) \cosh k_{0}(h-y)$, and in the edge condition $\pi \lambda$ is replaced by $\pi \lambda-i k_{0}\left(k_{0}-k\right) \sinh k_{0} h$.

The velocity potential in this case after using the results of Section 5 is given by

$$
\begin{aligned}
\phi(x, y)= & \cosh k_{0}(h-y) e^{-i k_{0} x}+A_{0} \cosh k_{0}(h-y) e^{i k_{0} x} \\
& +\sum_{n=1}^{\infty} A_{n} \cos k_{n}(h-y) e^{-k_{n} x}, \quad x>0,
\end{aligned}
$$

where

$$
\begin{aligned}
& A_{0}=\frac{k_{0}-k}{k_{0}+k}+\frac{4 i \pi M k_{0}^{2} \lambda\left(1+M k_{0}^{2}\right) \sinh k_{0} h}{K\left(k_{0}+k\right)\left\{2 k_{0} h\left(1+M k_{0}^{2}\right)+\left(1+3 M k_{0}^{2}\right) \sinh 2 k_{0} h\right\}} \\
& A_{n}=-\frac{4 \pi M k_{n}^{2} \lambda\left(1-M k_{n}^{2}\right) \sin k_{n} h}{K\left(k_{n}-i k\right)\left\{2 k_{n} h\left(1-M k_{n}^{2}\right)+\left(1-3 M k_{n}^{2}\right) \sin 2 k_{n} h\right\}} .
\end{aligned}
$$

When there is no surface tension the result agrees fully with the results of Chakrabarti and Sahoo [2]. Also when $k=0=M$, it is easily checked that $A_{0}=1$ and $A_{n}=0$ verifying the known results that the incident wave gets fully reflected back.

\section{Scattering of water waves by a vertical porous plate}

As an application of the solution of the two-sided wave maker problem as well as the problem of reflection of water waves by the vertical porous wall discussed 
above, in Sections 4 and 6, let us consider the problem of scattering of water waves by a vertical porous plate. The case of water of infinite depth has been discussed by Rhodes-Robinson [17]. Here, we will only discuss the case of water of finite depth. In this case, as in Section 2, the conditions (2.3), (2.4) and (2.5) are replaced by

$$
\phi_{j x}=-i k\left(\phi_{1}-\phi_{2}\right) \quad \text { on } \quad x=0,
$$

with the conditions at infinity given by

$$
\begin{aligned}
& \phi_{1} \rightarrow \cosh k_{0}(h-y)\left\{e^{-i k_{0} x}+A_{0} e^{i k_{0} x}\right\} \text { as } x \rightarrow \infty, \\
& \phi_{2} \rightarrow \cosh k_{0}(h-y) B_{0} e^{-i k_{0} x} \quad \text { as } x \rightarrow-\infty
\end{aligned}
$$

and

$$
\phi_{j y}=0 \quad \text { on } y=h,
$$

where $A_{0}$ and $B_{0}$ are the reflection and transmission coefficients to be determined.

The velocity potentials $\phi_{1}$ and $\phi_{2}$ are expressed in the forms as in the case of Section 4 , as given by

$$
\begin{aligned}
& \phi_{1}(x, y)=\cosh k_{0}(h-y)\left\{e^{-i k_{0} x}+A_{0} e^{i k_{0} x}\right\}+\sum_{n=1}^{\infty} A_{n} \cos k_{n}(h-y) e^{-k_{n} x}, x>0 \\
& \phi_{2}(x, y)=B_{0} \cosh k_{0}(h-y) e^{-i k_{0} x}+\sum_{n=1}^{\infty} B_{n} \cos k_{n}(h-y) e^{k_{n} x}, \quad x<0
\end{aligned}
$$

with

$$
\begin{aligned}
A_{0}+B_{0}= & 1+\frac{4 k_{0} M i C_{2}\left(1+M k_{0}^{2}\right) \sinh k_{0} h}{K\left\{2 k_{0} h\left(1+M k_{0}^{2}\right)+\left(1+3 M k_{0}^{2}\right) \sinh 2 k_{0} h\right\}}, \\
A_{n}+B_{n}= & \frac{4 k_{n} M\left(1-M k_{n}^{2}\right) i C_{2} \sin k_{n} h}{K\left\{2 k_{n} h\left(1-M k_{n}^{2}\right)+\left(1-3 M k_{n}^{2}\right) \sin 2 k_{n} h\right\}}, \\
& \text { for } n=1,2, \ldots,
\end{aligned}
$$

where $C_{2}$ is an arbitrary constant.

The full solution will be obtained once the unknown constants are determined completely.

Setting $\Phi(x, y)=\phi_{1}(x, y)-\phi_{2}(-x, y)$ and $\Psi(x, y)=\phi_{1}(x, y)+\phi_{2}(-x, y)$, we obtain two boundary-value problems for $\Phi(x, y)$ and $\Psi(x, y)$ which are particular cases of the problem already discussed in Section 6 with certain changes as described below:

The condition (6.1) is replaced by

$$
\Phi_{x}+2 i k \Phi=0 \quad \text { on } \quad x=0
$$


and

$$
\Psi_{x}=0 \text { on } \quad x=0
$$

In the infinity condition (6.2), $A_{0}$ is replaced by $A_{0}-B_{0}$ and $A_{0}+B_{0}$ in the problems for $\Phi(x, y)$ and $\Psi(x, y)$ respectively.

Finally, the edge condition (5.6) is replaced by

$$
\Phi_{x y}+2 i k \Phi_{y}=\pi\left(\lambda_{0}^{+}-\lambda_{0}^{-}\right), \quad \text { at } \quad(x, y)=(0+, 0)
$$

and

$$
\Psi_{x y}=\pi\left(\lambda_{0}^{+}+\lambda_{0}^{-}\right), \quad \text { at } \quad(x, y)=(0+, 0)
$$

Substituting for the expressions $\phi_{1}(x, y)$ and $\phi_{2}(x, y)$ from (7.3) and (7.4) in the relation for $\Phi(x, y)$ and $\Psi(x, y)$ and using the results of Section 6 , we obtain

$$
\begin{array}{r}
\Phi(x, y)=\cosh k_{0}(h-y)\left\{e^{-i k_{0} x}+\left(A_{0}-B_{0}\right) e^{i k_{0} x}\right\} \\
+\sum_{n=1}^{\infty}\left(A_{n}-B_{n}\right) e^{-k_{n} x} \cos k_{n}(h-y), \\
\Psi(x, y)=\cosh k_{0}(h-y)\left\{e^{-i k_{0} x}+\left(A_{0}+B_{0}\right) e^{i k_{0} x}\right\} \\
+\sum_{n=1}^{\infty}\left(A_{n}+B_{n}\right) e^{-k_{n} x} \cos k_{n}(h-y),
\end{array}
$$

with

$$
\begin{aligned}
A_{0}-B_{0}= & \frac{k_{0}-2 k}{k_{0}+2 k}+\frac{4 i \pi\left(\lambda_{0}^{+}+\lambda_{0}^{-}\right) M k_{0} \cosh k_{0} h}{\left(k_{0}+2 k\right)\left\{2 k_{0} h\left(1+M k_{0}^{2}\right)+\left(1+3 M k_{0}^{2}\right) \sinh 2 k_{0} h\right\}}, \\
A_{n}-B_{n}= & \frac{4 \pi M k_{n}\left(\lambda_{0}^{+}+\lambda_{0}^{-}\right) \cos k_{n} h}{\left(k_{n}-2 i k\right)\left\{2 k_{n} h\left(1-M k_{n}^{2}\right)+\left(1-3 M k_{n}^{2}\right) \sin 2 k_{n} h\right\}} \\
& \text { for } n=1,2, \ldots, \\
A_{0}+B_{0}= & 1+\frac{4 i \pi\left(\lambda_{0}^{+}-\lambda_{0}^{-}\right) M \cosh k_{0} h}{\left\{2 k_{0} h\left(1+M k_{0}^{2}\right)+\left(1+3 M k_{0}^{2}\right) \sinh 2 k_{0} h\right\}}
\end{aligned}
$$

and

$$
A_{n}+B_{n}=\frac{4 \pi M\left(\lambda_{0}^{+}-\lambda_{0}^{-}\right) \cos k_{n} h}{\left\{2 k_{n} h\left(1-M k_{n}^{2}\right)+\left(1-3 M k_{n}^{2}\right) \sin 2 k_{n} h\right\}}, \quad \text { for } \quad n=1,2, \ldots
$$


From the relations (7.12)-(7.15), we derive

$$
\begin{aligned}
& A_{0}=\frac{k_{0}}{k_{0}+2 k}+\frac{4 i \pi M\left[\left(k_{0}+k\right) \lambda_{0}^{+}-k \lambda_{0}^{-}\right] \cosh k_{0} h}{\left(k_{0}+2 k\right)\left\{2 k_{0} h\left(1+M k_{0}^{2}\right)+\left(1+3 M k_{0}^{2}\right) \sinh 2 k_{0} h\right\}} \\
& B_{0}=\frac{2 k}{k_{0}+2 k}+\frac{4 i \pi M\left[k \lambda_{0}^{+}-\left(k_{0}+k\right) \lambda_{0}^{-}\right] \cosh k_{0} h}{\left(k_{0}+2 k\right)\left\{2 k_{0} h\left(1+M k_{0}^{2}\right)+\left(1+3 M k_{0}^{2}\right) \sinh 2 k_{0} h\right\}}, \\
& A_{n}=\frac{4 M \pi\left[\left(k_{n}-i k\right) \lambda_{0}^{+}+i k \lambda_{0}^{-}\right] \cos k_{n} h}{\left(k_{n}-2 i k\right)\left\{2 k_{n} h\left(1-M k_{n}^{2}\right)+\left(1-3 M k_{n}^{2}\right) \sin 2 k_{n} h\right\}}, \text { for } n=1,2, \ldots
\end{aligned}
$$

and

$$
B_{n}=\frac{4 M \pi\left[i k \lambda_{0}^{+}+\left(k_{n}-i k\right) \lambda_{0}^{-}\right] \cos k_{n} h}{\left(k_{n}-2 i k\right)\left\{2 k_{n} h\left(1-M k_{n}^{2}\right)+\left(1-3 M k_{n}^{2}\right) \sin 2 k_{n} h\right\}}, \quad \text { for } \quad n=1,2, \ldots
$$

Substituting for $A_{n}, B_{n}$ for $n=0,1,2, \ldots$, in the expressions (7.3) and (7.4) for $\phi_{1}(x, y)$ and $\phi_{2}(x, y)$ we obtain the full solution in this case.

From the relations (7.5) and (7.14) we obtain $C_{2}=\pi\left(\lambda_{0}^{+}-\lambda_{0}^{-}\right)$. Also it is observed that the reflection and transmission coefficients depend on the edge slope constants on both sides of the wall.

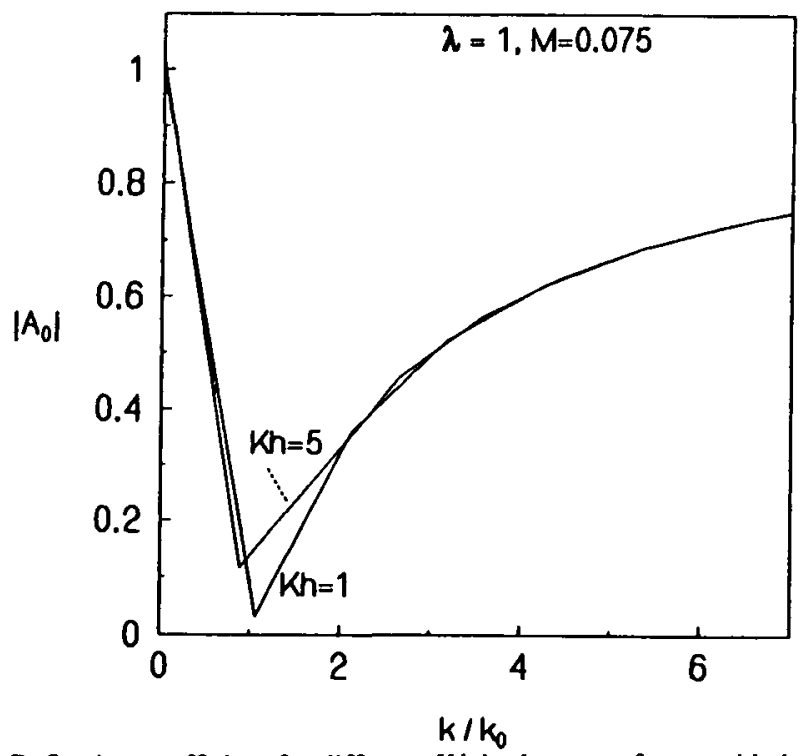

FIGURE 1. Reflection coefficient for different $K h$ in the case of a one-sided porous wall. 


\section{Numerical results and discussions}

Certain numerical results are determined in the case of water of finite depth by way of computing the wave amplitudes at large distances in the cases of scattering of water waves by a vertical porous plate as well as in the case of reflection of water waves by a vertical porous wall. Though in the formulation of the problem, we have assumed that $\lambda$ is an undeterminable constant and the full solution depends on this constant, in order to have certain observations about the effect of surface tension on the porous wall, the values of reflection coefficients versus the non-dimensional porosity factors have been plotted in Figure 1, for two different values of the wave effect parameter $K h$ (see Chwang [4]) for a fixed values of the edge slope constant $\lambda$ and the surface tension parameter $M$. It is observed that for large values of the porosity factors, the reflection coefficients coincide in both the cases. In Figure 2, the reflection coefficients versus the porosity factors have been plotted with and without the effect of surface tension. Here, also after a certain stage in both the cases, the reflection coefficients coincide. Finally in Figure 3, the reflection and transmission coefficients versus the porosity factor are plotted keeping the edge slope constants, the surface tension parameter as well as the wave effect parameter fixed in the case of scattering of water waves by vertical porous plate as has been discussed in Section 7.

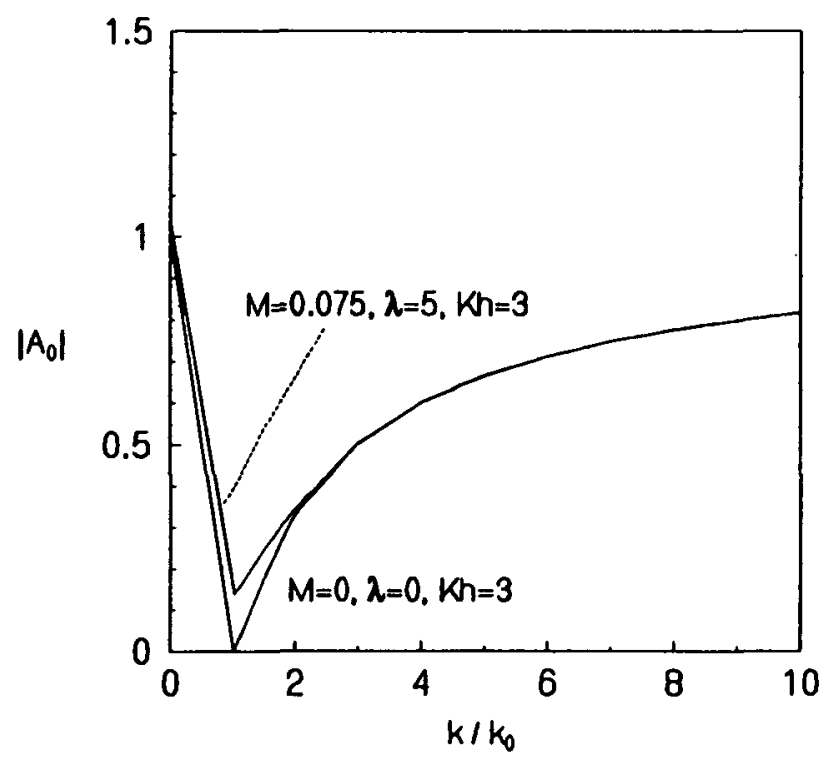

FIGURE 2. Reflection coefficient in the case of a one-sided porous wall with and without surface tension. 


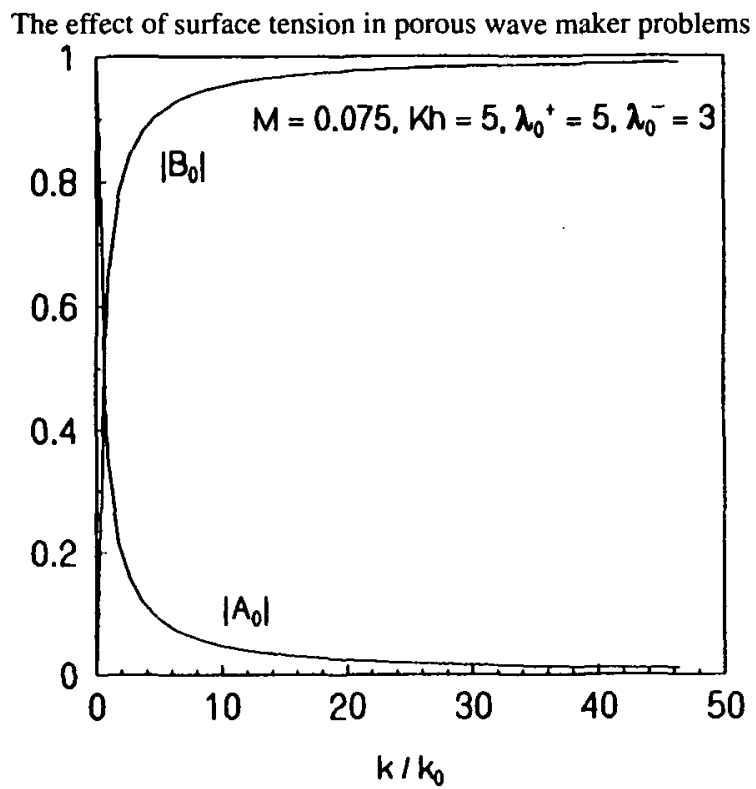

\section{Conclusion}

The problems of forced surface waves generated by a harmonically oscillating porous wave maker immersed in a fluid in the presence of surface tension have been studied by way of solving certain boundary-value problems associated with the two-dimensional Laplace equation. Both the cases of water of infinite as well as of finite depths are considered. In the case of water of infinite depth, a generalised mixed Fourier type transform has been employed to handle the boundary-value problems and in the case of water of finite depth, a generalisation of the eigenfunction expansion method has been exploited. As application of the general problems, the problem of reflection of water waves by a porous wall as well as the scattering by a porous plate have been considered in the case of water of finite depth. It can be noted that the results for the two-sided wave maker problems can be obtained from the one-sided wave maker problem by applying the reduction procedure adopted by Rhodes-Robinson [17] as well as by the procedure discussed in Section 7.

\section{Acknowledgement}

The authors are thankful to the anonymous referees for their comments and suggestions to improve the paper and to bring it into its present form. TS acknowledges the University Grants Commission, New Delhi for the financial support received as 
a research student of Indian Institute of Science, Bangalore. TS also acknowledges CSIR, New Delhi for financial support during the period when the paper was revised.

\section{References}

[1] A. Chakrabarti, "A note on the porous-wave maker problem", Acta Mechanica 77 (1989) 121-129.

[2] A. Chakrabarti and T. Sahoo, "Reflection of water waves by a nearly vertical porous wall", $J$. Austral. Math. Soc. Series B 37 (1996) 417-429.

[3] A. Chakrabarti and T. Sahoo, "Reflection of water waves in the presence of surface tension by a nearly vertical porous wall", J. Austral. Math. Soc. Series B 39 (1998) 308-317.

[4] A. T. Chwang, "A porous wave maker theory", J. Fluid Mech. 132 (1983) 395-406.

[5] M. A. Gorgui, M. S. Faltas and A. Z. Ahmed, "Capillary gravity waves in the presence of infinite porous plates", Il Nuovo Cimento, 15D, (1993) 793-808.

[6] T. H. Havelock, "Forced surface waves on water", Phil. Mag. 8 (1929) 569-576.

[7] L. M. Hocking, "Capillary gravity waves produced by a heaving body", J. Fluid Mech. 186 (1987) 337-349.

[8] L. M. Hocking and D. Mahdmina, "Capillary gravity waves produced by a wave maker", J. Fluid Mech. 224 (1991) 217-226.

[9] O. S. Madsen, "Waves generated by a piston type wave maker", Proc. 12th Coastal Engng Conf., ASCE (1970), pp. 589-607.

[10] B. N. Mandal and S. Bandyopadhyay, "A note on the plane vertical wave maker in the presence of surface tension”, Q. Appl. Math. XLIX, (1991) 627-633.

[11] B. N. Mandal and A. Chakrabarti, "The plane vertical wave maker problem - revisited", Appl. Math. Lett. 1, (1988) 255-258.

[12] P. F. Rhodes-Robinson, "On the forced surface waves due to a vertical wave maker in the presence of surface tension", Proc. Camb. Phil. Soc. 76 (1971) 323-337.

[13] P. F. Rhodes-Robinson, "On surface waves in the presence of immersed vertical boundaries I", $Q$. Jour. Mech. and Appl. Math. XXXII, (1979) 109-124.

[14] P. F. Rhodes-Robinson, "On surface waves in the presence of immersed vertical boundaries II", $Q$. Jour. Mech. and Appl. Math. XXXII, (1979) 125-133.

[15] P. F. Rhodes-Robinson, "On waves at an interface between two liquids", Math. Proc. Camb. Phil. Soc. 88 (1980) 183-191.

[16] P. F. Rhodes-Robinson, "Note on the reflection of water waves at a wall in the presence of surface tension", Proc. Camb. Phil. Soc. 92 (1982) 369-373.

[17] P. F. Rhodes-Robinson, "On waves in the presence of vertical porous boundaries", J. Austral. Math. Soc. Series B 39 (1997) 104-120.

[18] W. E. Williams, "Diffraction of surface waves on an incompressible fluid", J. Fluid Mech. 22 (1065) ग52-356 